\title{
A Method for Analysis of Electrophysiological Responses Obtained from the Motor Fibers of the Human Internal Capsule
}

\author{
E.G. Duerden ${ }^{1}$, K.W. Finnis ${ }^{2}$, T.M. Peters ${ }^{2}$, and A.F. Sadikot ${ }^{1}$ \\ ${ }^{1}$ Montreal Neurological Institute, Division of Neurosurgery \\ 3801 University St. Montreal, QC, Canada, H3A 2B4 \\ ${ }^{2}$ Robarts Research Institute \\ 100 Perth Drive, London, ON, Canada, N6A 5K8 \\ sadikotabic.mni.mcgill.ca
}

\begin{abstract}
During stereotactic surgery in the ventrolateral (VL) thalamic nucleus we localized the motor fibers of the internal capsule (IC) by electrophysiological stimulation. Knowledge of the location and motor somatotopy in the IC helps predict the boundaries of the VL. Although the somatotopy of the IC in individual patients is determined during their respective surgeries, quantitative analysis of somatotopic data from multiple subjects within a common magnetic resonance imaging (MRI) reference space has not been attempted. Here we describe a method for group analysis, in a single coordinate system, of responses obtained from the IC adjacent to the thalamus. We analyzed tongue, face, arm, and leg motor responses in 25 cases of thalamotomy. We used existing software to develop a three dimensional (3D) database of motor responses. Our preliminary analysis suggests a face-anterior to leg-posterior somototopic organization, with some overlap between adjacent representations.
\end{abstract}

\section{Introduction}

Thalamotomy and thalamic deep brain stimulation (DBS) are proven treatments for the relief of tremor associated with Parkinson's disease (PD) and Essential tremor $[1,2,3]$. Target localization for these procedures is achieved using magnetic resonance imaging (MRI), ventriculography or computed tomography (CT). As an additional measure, the target region is confirmed intraoperatively by recording and/or stimulating in thalamic nuclei and/or the internal capsule (IC) to determine tissue function and map somatotopy. Knowledge of the borders and the somatotopic organization of the IC assists in predicting the location of the ventral lateral (VL) nucleus, the region targeted for thalamotomy and thalamic DBS for tremor. At present, the somatotopic organization of the IC remains unclear [4,5]. According to classical anatomical studies, the face and eye representation lies in the anterior limb, the tongue representation lies in the genu, and the arm and leg representations lie in the posterior limb [6]. Several functional atlases of the IC created from 
electrophysiological data from movement disorder surgery have shown that the somatotopic organization of the IC is inconsistent with the classical view $[7,8,9]$. These studies demonstrated that the motor fibers were actually located in the middle third of the posterior limb and contain a complete face, arm, and leg representation $[7,8,10]$. Bertrand et al. $[7,8]$ used a curved retractable electrode to obtain direct responses from the IC at the level of the thalamus. The authors then displayed electrophysiological responses in a two-dimensional (2D) atlas slice derived from the Schaltenbrand and Bailey atlas [11]. Using the midcommissural plane (MCP) as a reference point and applying corrections for varying third ventricle widths, they reported that the representation of the face was anterior to the arm, which in turn was anterior to the representation of the leg. They reported a considerable amount of overlap between body part representations in both individuals and on group analysis. Tasker at al. [9] displayed data on 2D atlas slices [11] scaled to match the length of each patient's intercommissural line. These authors used current spread from a straight electrode to the adjacent IC. Several data points were located outside the area prescribed by the atlas to be the IC, extending into the atlas-defined territory of the thalamus. This was attributed to inter-subject anatomical differences in the location of the IC and the width of the third ventricle. The authors found little evidence of somatotopic organization of the motor fibers.

These early studies provided the basis for the understanding of the organization of the posterior limb of the IC in humans. However, the information describing the exact localization of body part representations is limited by the linear scaling techniques employed to register the electrophysiology to the standardized anatomical atlas. By plotting 3D data on 2D atlas slices using standardized calculations to accommodate for anatomical variability (or plotting data relative to other anatomical structures) the data points can only approximate the anatomy from which they were obtained in the patient brain. As a result, clustering of patient data points within the coordinate system of a 2D atlas is difficult to analyze and unsuitable for application to surgical planning. Another drawback of the early functional atlases is their reliance on 2D printed stereotactic atlases of anatomy [12]. These atlases were constructed from one individual, or from the hemispheres of several individuals, and cannot be considered representative of the general population. During the construction of these anatomical atlases, it was not uncommon for several slices to shrink or be damaged during histochemical processing. As a result, uneven inter-slice distances were often present in the final printed version of the atlas. These gaps make accurate annotation of 3D data points to the atlas anatomy nearly impossible. Furthermore, by displaying functional data in only $2 \mathrm{D}$ horizontal planes this method does not present the dynamic organization of the motor fibers as they course through the IC in a rostrocaudal direction [13]. Finally, representation of functional data using only the coordinate system of an anatomical atlas, rather than a volumetric MRI further limits the utility of this approach. The ability to represent data from multiple subjects in a common MRI reference space would be advantageous to understanding brain organization in human subjects, and potentially allow for applicability of these electrophysiological atlases during surgery in deep brain structures.

Recently, Finnis et al. [14,15,16] developed surgical guidance software, collectively referred to as ASP (Atamai Surgical Planner), that was used to develop a functional atlas of electrophysiological responses obtained during movement disorder surgery. 
Data from the sensorimotor thalamus, globus pallidus internus, and subthalamic nucleus were incorporated into this atlas. This electrophysiological atlas has overcome limitations of previous functional atlases in several ways. In particular, the atlas can account for inter-subject anatomical variability through the use of a nonlinear registration technique that transfers the raw electrophysiological data into a standard brain MRI volume that serves as a common coordinate system for all atlas data. The atlas may be nonlinearly registered to a patient's preoperative MRI to aid in surgical guidance and target localization.

As a further application of this work, we reassessed the somatotopic organization and the degree of somatotopic overlap between body part representations in the posterior limb of the IC using the ASP environment. Electrophysiological data were obtained from within the posterior limb of the $\mathrm{IC}$, the region containing projections from the primary motor cortex [13]. We explored the tongue, face, arm, and leg representations using the electrophysiological data from 25 thalamotomies. A diseasebased 3D electrophysiological database that describes the representation of these body parts within the IC was developed. When completed, this atlas of motor responses in the IC can be registered to a patient's preoperative MRI to aid in target localization and surgical planning of VL thalamotomy and DBS surgery.

\section{Materials and Methods}

\subsection{Patients}

The patients included in the study were operated on between 1995 and 2002 by the same surgeon (A.F.S) at the Montreal Neurological Hospital and Institute (MNI) Montreal, Quebec, Canada (Table 1). All patients underwent surgery for tremor and were examined by a neurologist and neurosurgeon prior to surgery. There were a total of 24 patients who underwent 25 thalamotomy procedures (17 males, 7 females; mean age $=58$ years, $\operatorname{stdev}=13.7$ years $)$. Nine lesions were made on the right, and 16 were made on the left (Table 1). Patients were selected based on the availability and quality of their preoperative MRIs. Those patients with limb amputations, tumors, lesions or other pathology that might affect somatotopic organization or hinder the nonlinear registration process were excluded from the study.

Table 1. Patient Characteristics

\begin{tabular}{clccc} 
Subjects & Procedure & Gender & Age & $\begin{array}{c}\text { Third Ventricle } \\
\text { Width (mm) }\end{array}$ \\
\hline \hline 24 & Left Thalamotomies, 16 & Males, 17 & $58( \pm 13.7)$ & $4.95( \pm 1.9)$ \\
& Right Thalamotomies, 9 & Females, 7 & &
\end{tabular}




\subsection{Imaging}

Patient images were acquired using a 1.5T Phillips ACS-2 MRI Scanner using a 3D gradient echo sequence with TR: $27 \mathrm{~ms}$, TE: $9 \mathrm{~ms}$, Nex:2 and flip angle of 30 degrees was used to produce a volume with $1 \mathrm{~mm} \times 1 \mathrm{~mm} \times 1.5 \mathrm{~mm}$ resolution.

\subsection{Surgical Procedure}

Before the surgical procedure a local anesthetic was applied. This was followed by the application of the Olivier Bertrand Tipal (OBT) (Tipal Instruments, Quebec) stereotactic head frame. Target localization was based on stereotactic MR imaging (MRI) and ventriculography, and confirmed using intraoperative physiological mapping. The motor responses of the IC and the sensory cutaneous responses from the ventral posterior thalamic nucleus were mapped, in order to localize the optimal target in the VL nucleus, as described in our previous publications [1,17]. A digitized version of the Scaltenbrand and Wahren atlas [12] was integrated with the patient's MRI scan in each case using an automated procedure as previously described. In each case the target chosen for lesioning was centered on the posterior part of the VL.

Electrical stimulation was administered using a stereotactically directed insulated curved, retractable macroelectrode (Figure 1). This design allows the tip of the probe to be extended at $1 \mathrm{~mm}$ intervals and permits a larger volume of tissue to be mapped in a single trajectory. When localizing motor responses in the IC, stimulation was administered between $0.25 \mathrm{~V}$ and $2 \mathrm{~V}$ for $2 \mathrm{msec}$ duration at $60 \mathrm{~Hz}$. Responses obtained at the lowest thresholds $(0.25 \mathrm{~V}$ and $0.5 \mathrm{~V})$ were selected for

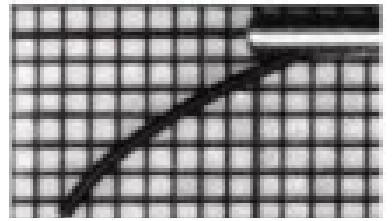

Fig. 1. Retractable curved electrode. (scale: $1 \mathrm{~mm}$ ) analyses. Current spread generated at these levels is limited, and therefore produces a more reliable and localized response. At each point of stimulation the stereotactic frame coordinates, declination and azimuth of the probe, and the degree to which the electrode was extended were recorded.

\subsection{Electrophysiology Database}

The electrophysiological database was created using the ASP platform $[14,15,16]$. Data points were tagged within each patient's preoperative MRI according to their original frame space coordinates. The patient's scan was then nonlinearly registered, using a previously validated image-matching algorithm (ANIMAL) [18], to a standard, high-resolution MR volume (CJH27) [19]. This standard volume was created from 27 registered T1-weighted images of the same individual, where each of 26 volumes was mapped to the 27 th using a rigid-body transformation. All 27 images were then averaged on a pixel basis. This target image serves as a common image space for all of the electrophysiological data obtained from patients. ANIMAL registers the patient MRI to the standard MRI by computing a nonlinear transform. The nonlinear algorithm produces a 3D deformation grid that describes a one-to-one mapping of the patient image to the standard image. The grid created for each patient image is used to register the patients' functional data into the image space of the standard MRI. 
To determine the efficacy of the nonlinear algorithm, the anterior commissure (AC) and posterior commissure (PC) coordinates were first manually determined in the standard MRI. The AC and PC coordinates for all patients were plotted within the ASP interface. The points were then nonlinearly registered to the target image. The transformed coordinates of the points were then compared with those of the target image. The absolute differences and the mean Euclidean differences between the points were directly calculated (Table 2). All points found within a $3 \mathrm{~mm}$ distance from the AC-PC coordinates of the target image were included in this data set.

Table 2. ${ }^{\mathrm{a}}$ Mean locations of the AC-PC coordinates. ${ }^{\mathrm{b}}$ Mean difference of the coordinates from those of CJH27. ${ }^{\mathrm{c}}$ Maxium error. ${ }^{\mathrm{d}}$ Euclidean error

\begin{tabular}{ccccccc}
\hline & \multicolumn{3}{c}{$\mathrm{AC}$} & \multicolumn{3}{c}{$\mathrm{PC}$} \\
\hline \hline Mean & $\mathrm{X}$ & $\mathrm{y}$ & $\mathrm{Z}$ & $\mathrm{x}$ & $\mathrm{y}$ & $\mathrm{z}$ \\
\hline \hline Locations $^{\mathrm{a}}$ & $0.5( \pm 0.64)$ & $3.28( \pm 0.12)$ & $-4.58( \pm 1.24)$ & $0.13( \pm 0.78)$ & $-23.96( \pm 0.54)$ & $-2.57( \pm 1.16)$ \\
Difference $^{\mathrm{b}}$ & -0.45 & 0.12 & -0.42 & -0.03 & -0.04 & -0.43 \\
Max. Error $^{\mathrm{c}}$ & -2.06 & 1.43 & -2.8 & 2.5 & 1.12 & -2.54 \\
Error $^{\mathrm{d}}$ & & 0.63 & & & 0.43 &
\end{tabular}

\subsection{Entering Data and Standardization of Responses}

The entry of data into the ASP interface is described in detail elsewhere $[14,15,16]$. Functional data were annotated to the patient's MRI using a graphical user interface (GUI) incorporated into ASP. To facilitate coding of the type of response observed and the location on the body there is an anatomical model representing both dorsal and ventral views of one half of the body (Figure 2). The data were entered into the ASP interface by assigning a multi-parameter code to each point of stimulation. Each data point is assigned a patient number, trajectory number, stimulation intensity, a body part number, and the type of response elicited.

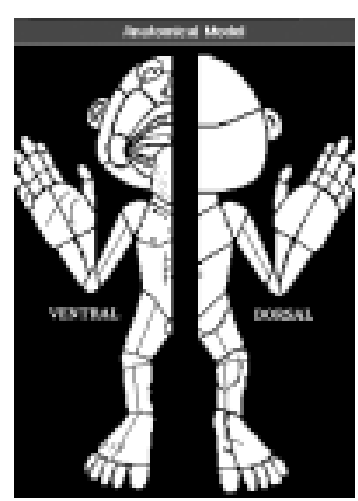

Fig. 2. Homunculus model used for entering data into and retrieving it from the database.

\subsection{Statistical Analysis}

Data from right and left procedures were analyzed together by transforming the medial-lateral $(\mathrm{x})$ coordinates to negative values (left side). We analyzed the data using discriminant analysis to test the null hypothesis that there is no separation between the four body part representations. To determine the average area of concentration of electrophysiology data points for each body part representation, we examined the center of mass (COM) coordinates (mean coordinates along the mediallateral (x), anterior-posterior (y), and superior-inferior (z) axes). To assess the null hypothesis of there being no difference between the COM coordinates along the $\mathrm{x}, \mathrm{y}$, 
and z-axes we performed an ANOVA on the COM coordinates for each of the tongue, face, arm, and leg representations. In order to assess the degree of overlap between body part representations we directly calculated the amount of overlapping responses that occurred for each representation.

\section{Results}

315 responses resulted in current-induced muscle contractions typically observed during stimulation of the IC . Of these, 66 responses resulted in contraction of the tongue, 86 the face, 106 the arm, and 57 the leg. Responses for each area maintained separate positions in the posterior limb of the IC (Wilkes' Lambda, $\mathrm{P}=0.0$ ).

Figure 3 shows the COM coordinates displayed within the CJH27 MRI registered with a digitized stereotactic brain atlas [17]. The COM coordinates for each representation showed that the tongue and face representation were located more anterior to the leg representation, and the arm representation was located in an intermediate position. The mean coordinates along the medial-lateral $\quad(\mathrm{x}) \quad(\mathrm{F}=3.41, \quad \mathrm{P}=0.018)$,

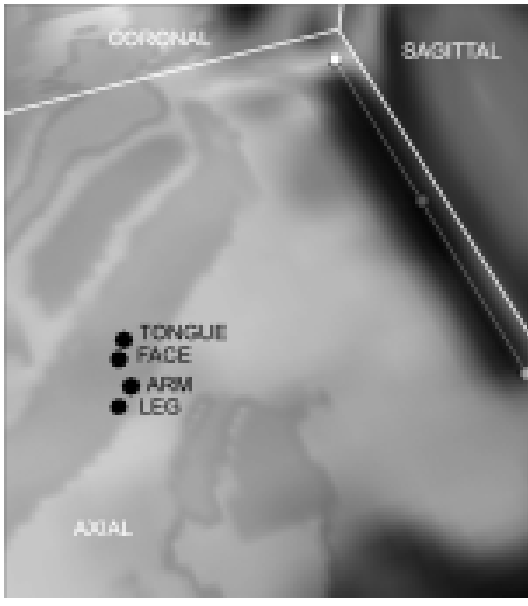

Fig. 3. Mean locations of body part representations. anterior-posterior $(\mathrm{y}),(\mathrm{F}=50.83, \mathrm{P}=0.0)$ and superior-inferior $(\mathrm{z}),(\mathrm{F}=9.41, \mathrm{P}=0.0)$ axes were statistically distinct. Results from post hoc testing are displayed in Table 3.

Table 3. Post hoc comparisons of $\mathrm{x}, \mathrm{y}$ and $\mathrm{z}$ coordinates for each body part representation (Tukey's HSD). * $=$ the mean difference is significant at the 0.05 level

\begin{tabular}{|c|c|c|c|c|c|c|c|}
\hline & & & \multicolumn{2}{|c|}{$\mathrm{X}$} & $\mathrm{Y}$ & \multicolumn{2}{|c|}{$\mathrm{Z}$} \\
\hline & & Difference & $\mathrm{p}$ & Difference & $\mathrm{p}$ & Difference & $\mathrm{p}$ \\
\hline \multirow[t]{3}{*}{ Face } & Tongue & 0.41 & 0.661 & -0.36 & 0.509 & $-1.31^{*}$ & 0.008 \\
\hline & Arm & 0.00 & 1.000 & $1.92 *$ & 0.000 & -0.13 & 0.985 \\
\hline & Leg & -0.83 & 0.121 & $2.29 *$ & 0.000 & 1.08 & 0.056 \\
\hline \multirow[t]{3}{*}{ Tongue } & Face & -0.41 & 0.661 & 0.36 & 0.509 & $1.31^{*}$ & 0.008 \\
\hline & Arm & -0.41 & 0.634 & $2.29 *$ & 0.000 & $1.19 *$ & 0.015 \\
\hline & Leg & $-1.24 *$ & 0.010 & $2.66^{*}$ & 0.000 & $2.39 *$ & 0.000 \\
\hline \multirow[t]{3}{*}{ Arm } & Face & 0.00 & 1.000 & $-1.92 *$ & 0.000 & 0.13 & 0.985 \\
\hline & Tongue & 0.41 & 0.634 & $-2.29 *$ & 0.000 & $-1.18^{*}$ & 0.015 \\
\hline & Leg & -0.83 & 0.098 & 0.37 & 0.494 & $1.21 *$ & 0.018 \\
\hline \multirow[t]{3}{*}{ Leg } & Face & 0.83 & 0.121 & $-2.29 *$ & 0.000 & -1.08 & 0.056 \\
\hline & Tongue & $1.24 *$ & 0.010 & $-2.66^{*}$ & 0.000 & $-2.39 *$ & 0.000 \\
\hline & Arm & 0.83 & 0.098 & -0.37 & 0.494 & $-1.21 *$ & 0.018 \\
\hline
\end{tabular}


The percentage of overlapping centroids at which point a response was elicited for more than one body part representation was determined. Of the 66 muscle contraction responses elicited from the tongue, 57.6\% (38 responses) overlapped the face responses, $15.2 \%$ (10 responses) overlapped with arm responses, while none of these data overlapped the leg responses. 86 responses were recorded from the face. Of these, $70.9 \%$ (61 responses) overlapped with the tongue responses, $30.2 \%$ (26 responses) with the arm responses, and 2.3\% (2 responses) with the leg responses. There were 106 arm responses, of which 10.4\% (11 responses) overlapped with tongue responses, $37.7 \%$ (40 responses) overlapped with face responses, and $34.2 \%$ (37 responses) overlapped with the leg responses. From 57 muscle contraction responses that were recorded from the leg, none overlapped with the tongue responses, 7\% (4 responses) overlapped with the face responses, and $50.9 \%$ (29 responses) of the total were found to overlap with the arm responses.

\section{Discussion}

The somatotopic organization of the human IC, due to its clinical significance, has long been a focus of great interest and debate. Whereas the homuncular representation of the primary motor cortex is well-defined as a result of direct cortical stimulation in awake patients [20], little information exists on the location and organization of motor fibers of the IC in humans. Here we developed a method examining the organization of the motor fibers of the IC by 3D representation of electrophysiological data from multiple subjects in a common image reference space. Using this method, our preliminary results suggest that projections from the primary motor cortex are localized to the posterior limb of the IC. With the help of the nonlinear registration techniques used to analyze the data, we were also able to quantify the amount of somatotopic overlap that exists between body part representations in multiple subjects. We report that although there is some overlap between body part representations, each one largely maintains a separate location in the IC suggesting preserved cortical somatotopy. An electrophysiological MRI integrated atlas of the functional organization of the IC will serve as an important guide during stereotactic neurosurgery. Our study contributes important knowledge about the position and organization of motor fibers in the human IC, and allows for better interpretation of functional deficits seen on imaging studies after stroke or other brain pathologies.

Acknowledgements. This work was supported by the Canadian Institute for Health Research, grant MT11540. The authors thank Dr. Yves Starreveld and Dr. David Gobbi (Robarts Research Institute) for assistance with software development; Fabrice Rouah, Christopher Grindrod, and Dr. Keith Worsley (McGill University) for their input on statistical analyses.

\section{References}

1. Atkinson, J. D., Collins, D. L., Bertrand, G., Peters, T. M., Pike, G. B., and Sadikot, A. F.: Optimal location of thalamotomy lesions for tremor associated with Parkinson disease: a probabilistic analysis based on postoperative magnetic resonance imaging and an integrated digital atlas. J. Neurosurg. 96 (2002) 854-866 
2. Koller, W. C., Pahwa, P. R., Lyons, K. E., and Wilkinson, S. B.: Deep brain stimulation of the Vim nucleus of the thalamus for the treatment of tremor. Neurology 55 (2000) S29S33

3. Ohye, C.: Use of selective thalamotomy for various kinds of movement disorder, based on basic studies. Stereotact. Funct. Neurosurg. 75 (2000) 54-65

4. Fries, W., Danek, A., Scheidtmann, K., and Hamburger, C.: Motor recovery following capsular stroke. Role of descending pathways from multiple motor areas. Brain 116 ( Pt 2) (1993) 369-382

5. Misra, U. K. and Kalita, J.: Central motor conduction studies in internal capsule and corona adiata infarction. J. Neurol. 244 (1997) 579-585

6. Foerster, O.: Motorishce Felder und Bahnen. In: Bomke, O., Foerster, O. (eds.): Handbuch der Neurologie. Springer, Berlin (1936) 1-357

7. Bertrand, G., Blundell, J., and Musella, R.: Stimulation during stereotactic operations for dyskinesias. J. Neurosurg. 24 (1966) 419-423

8. Hardy, T. L., Bertrand, G., and Thompson, C. J.: The position and organization of motor fibers in the internal capsule found during stereotactic surgery. Appl. Neurophysiol. 42 (1979) 160-170

9. Tasker, R. R., Organ, L. W., and Hawrylyshyn, P. A.: The Thalamus and Midbrain of Man. Carles C. Thomas, Springfield (1982)

10. Guiot, G., Sachs, M., Hertzog, E., Brion, S., Rougerie, J., Dallouz, J., and Napoleone, F.: Stimulation électrique et lésions chirurgicals de la capsule interne: déductions anatomiques et physiologiques. Neuro-Chirurgie 5 (1959) 17-42

11. Schaltenbrand, G. and Bailey, P.: An Introduction to Stereotaxis with an Atlas of the Human Brain. Thieme, Stuttgart (1959)

12. Schaltenbrand, G. and Wahren, P.: Introduction to stereotaxis with an atlas of the human brain. Georg Thieme Verlag, Stuttgart (1977)

13. Ross, E. D.: Localization of the pyramidal tract in the internal capsule by whole brain dissection. Neurology 30 (1980) 59-64

14. Finnis, K. W., Starreveld, Y. P., Parrent, A. G., Sadikot, A. F., and Peters, T. M.: Threedimensional database of subcortical electrophysiology for image-guided stereotactic functional neurosurgery. IEEE Trans. Med. Imaging 22 (2003) 93-104

15. Finnis, K. W., Starreveld, Y. P., Parrent, A. G., Sadikot, A. F., and Peters, T. M.: Application of a population based electrophysiological database to the planning and guidance of deep brain stereotactic neurosurgery. In: Dohi, T., Kikinis, R. (eds.): MICCAI 2002, LNCS 2489, (2002) 69-76

16. Finnis, K. W., Starreveld, Y. P., Parrent, A. G., and Peters, T. M.: A 3-dimensional database of deep brain functional anatomy and its application to image-guided neurosurgery. In: Dohi, T., Kikinis, R. (eds.): MICCAI 2000, LNCS 2489 (2000) 1-8

17. St Jean, P., Sadikot, A. F., Collins, L., Clonda, D., Kasrai, R., Evans, A. C., and Peters, T. M.: Automated atlas integration and interactive three-dimensional visualization tools for planning and guidance in functional neurosurgery. IEEE Trans. Med. Imaging 17 (1998) 672-680

18. Collins, D. L., Holmes, C. J., Peters, T. M., and Evans, A. C.: Automated 3-D modelbased neuroanatomical segmentation. Human Brain Mapping 3 (1995) 190-208

19. Holmes, C. J., Hoge, R., Collins, D. L., Woods, R., Toga, A. W., and Evans, A. C.: enhancement of MR images using registration for signal averaging. Journal of Assisted Computed Tomography 22 (1998) 324-333

20. Penfield, W. and Rassmussen, T.: The Cerebral Cortex of Man. MacMillan,New York (1950). 